\title{
Análisis de Parámetros Objetivos y Subjetivos en Pre-Amplificadores de Audio
}

\author{
Analysis of Objective and Subjective Parameters \\ in Pre-Amplifiers Audio
}

\section{Beltrán Bríñez Javier A. ${ }^{1}$, Marcela Mejía Cuevas ${ }^{2}$, Manuel Torres ${ }^{3}$}

Facultad de Ingeniería, Universidad de San Buenaventura, Bogotá, Colombia

\author{
${ }^{1}$ jabb-k@hotmail.com \\ ${ }^{2}$ marcelam123ehotmail.com \\ ${ }^{3}$ mtorres@usbbog.edu.co
}

Recibido: 6/05/2013 • Aprobado: 02/07/2013

\section{RESUMEN}

El siguiente artículo explica los resultados de las mediciones en cinco (5) diferentes pre-amplificadores, entre estado sólido y válvulas al vacío, de parámetros objetivos de THD -Análisis de Fourier- y función de transferencia, y parámetros subjetivos de calidez y brillo, en una muestra no probabilística de expertos con ingenieros de grabación que cuentan con una experiencia entre quince (15) y veinticinco (25) años, con el fin de encontrar una correspondencia directa entre estos parámetros y determinar qué elementos electrónicos están relacionados con la percepción auditiva de calidez y brillo en una grabación realizada con estos dispositivos.

Palabras clave: banco de pruebas, brillo, calidez, función de transferencia, pre-amplificador, THD - Análisis de Fourier.

\section{AbstRact}

This article explains the results of the measurements made on five (5) different pre-amplifiers, including solid state and vacuum tube, of objective parameters such as THD - Fourier Analysis- and Transfer Function and subjective parameters such as Warmness and Brightness evaluated on a nonrandom sample of experts conformed by recording engineers with professional experience between fifteen (15) and twenty five (25) years, in order to find a relation between these parameters and define which electronics elements are related with the aural perception of warmness and brightness in a recording made with these devices.

Keywords: testing bench, brightness, warmness, transfer function, pre-amplifier, THD -fourier analysis. 


\section{INTRODUCCIÓN}

Uno de los campos de estudio de la Ingeniería de Sonido es el desarrollo y la implementación de diferentes dispositivos electrónicos, encargados de realizar varios procesos en las señales de audio, buscando siempre manipularlas para poder lograr el resultado deseado. Uno de estos dispositivos es el amplificador de señal pequeña o comercialmente conocido como pre-amplificador. A lo largo del tiempo, se han implementado diferentes tipos de preamplificadores que varían en su diseño o en los elementos empleados en su construcción, con el fin de cumplir ciertos propósitos en diversos campos, entre otros, la grabación y producción de audio profesional.

Los primeros amplificadores de señal pequeña en implementarse fueron diseñados con válvulas al vacío, ya que algunas décadas atrás era el elemento que se conocía para lograr un circuito de amplificación de una señal eléctrica. Posteriormente, en los 60s, la invención del transistor hace posible que dichos circuitos se obtengan con mayor facilidad y economía. Sin embargo, las válvulas al vacío se siguen implementando actualmente por motivos tanto subjetivos (tales como la calidez y la suavidad Barbour [1] como objetivos, (THD, modelos psicoacústicos (Dobrucki, Maleczek, \& Maurycy, 2005) [2], que estas tienen sobre la señal procesada).

En este artículo se compara un banco de preamplificadores usando un tríodo dual 12AX7 con tres configuraciones de salida, una etapa de solo válvulas y dos con transformadores comerciales, construidos por los autores del mismo, con el fin de analizar los parámetros objetivos y subjetivos de las diferentes configuraciones para su posterior comparación entre ellos mismos y dos pre-amplificadores comerciales: el API512C de estado sólido y el Manley Voxbox de válvulas al vacío.

\section{Metodología}

\section{A. Técnicas de Recolección de Información}

1) Parámetros Objetivos. THD -Análisis de Fourier- McCarthy [3], se aplica una señal seno de $1 \mathrm{KHz}$ a un $1 \mathrm{Vmax}$ a los sistemas y con el analizador de espectro se analiza el contenido armónico, determinando la cantidad y amplitud de cada uno de estos. En la Fig. 1 se muestra un diagrama de bloques para la medición de THD -Análisis de Fourier-.

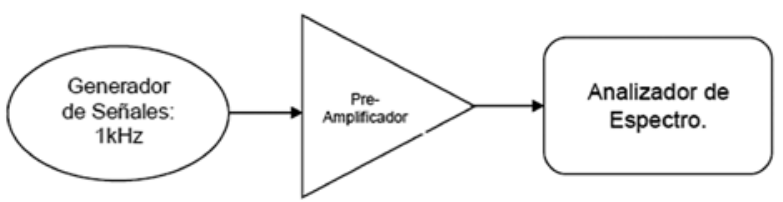

Fig. 1 Diagrama de bloques para la medición de THD -Análisis de Fourier-

Función de transferencia McCarthy [3], con ayuda de un software de análisis se registra el comportamiento en frecuencia tanto en amplitudes como en respuesta en fase de los sistemas, con el fin de determinar cuál de estos posee una mayor linealidad en el espectro audible. En la Fig. 2 se muestra un diagrama de bloques para la medición de función de transferencia.

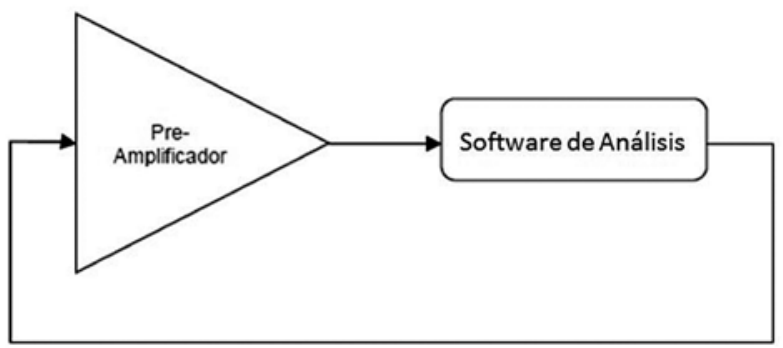

Fig. 2 Diagrama de bloques, para la medición de función de transferencia.

2) Parámetros Subjetivos. Se graba una composición musical con los siguientes instrumentos:

- Batería.

- Bajo.

- Teclado.

- Guitarra eléctrica con amplificador.

- Voz.

- Saxofón alto. 
Los instrumentos que se analizan son la guitarra eléctrica y la voz. La única variable de las muestras es el pre-amplificador seleccionado para el ensayo, manteniendo constante la toma de grabación y el micrófono para cada una de ellas, AKG C414.
Cada una de las muestras se registra en un DAW -Digital Audio Workstation, por canales independientes. La Fig. 3 muestra el diagrama de bloques del registro de las señales para las pruebas subjetivas.

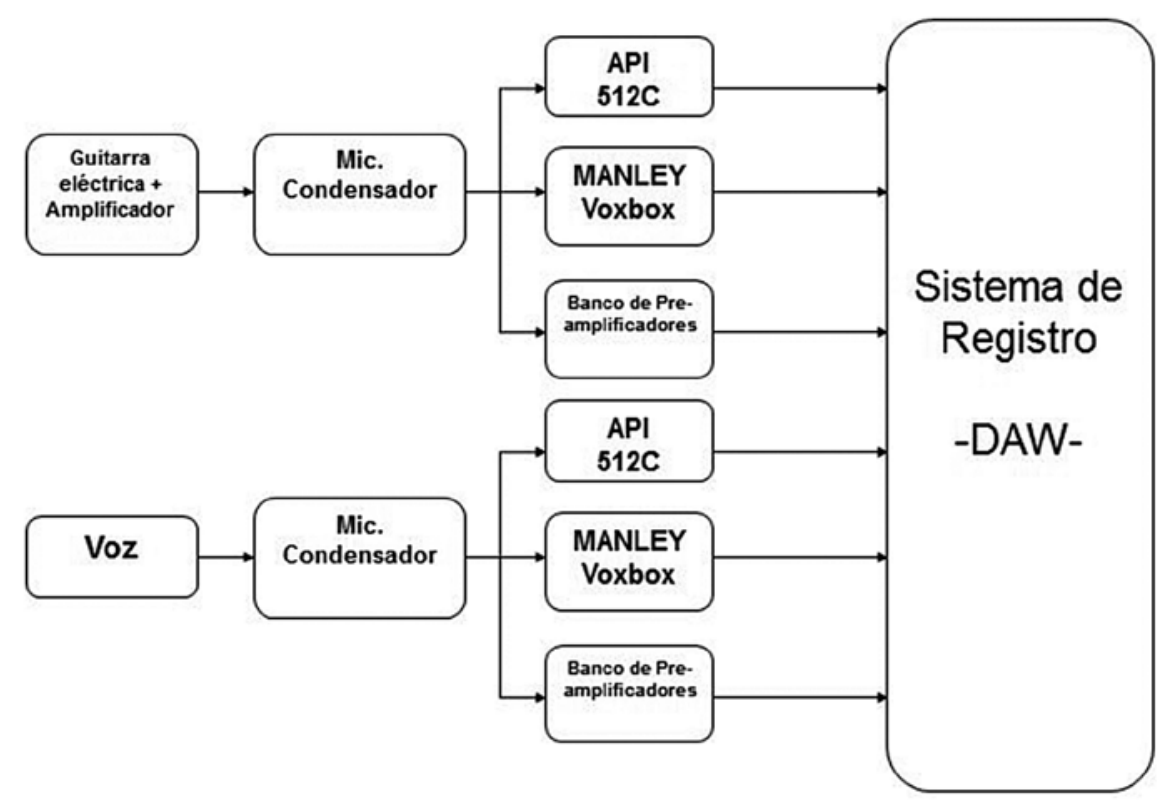

Fig. 3 Diagrama de bloques, registro de señal para pruebas subjetivas

Luego, cada una de las muestras se expone a la trumento registrado y la calidez y el brillo de los ampoblación sobre la cual se aplica una encuesta para plificadores seleccionados. La Fig. 4 muestra el diaevaluar los parámetros de calidad de sonido del ins- grama de bloques para la aplicación de la encuesta.

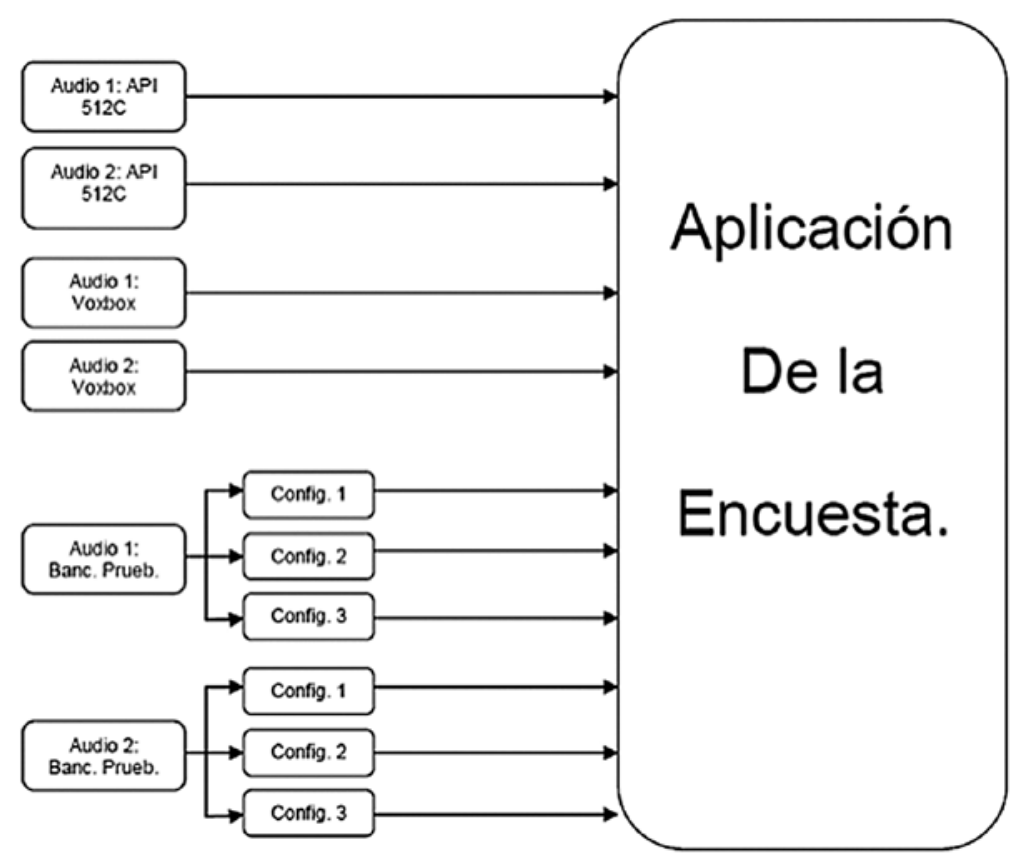

Fig. 4 Diagrama de bloques para aplicación de la encuesta. 
La encuesta por aplicar sigue dos modelos de escalamiento para determinar los diferentes parámetros que se han de evaluar. Para la evaluación de los parámetros de calidez (presencia de graves, bajos adecuados y buena relación de los armónicos fundamentales a los armónicos superiores) y brillo (énfasis en altas frecuencias y armónicos con gran presencia con respecto a los fundamentales) se aplica el escalograma de Guttman, siendo 1 la opción más cálida y 5 la más brillante, mientras la evaluación de la calidad de la señal sigue la escala de Likert (Hernández, Fernández, \& Baptista, [4]). Las Figs. 5 y 6 muestran el modelo de encuesta aplicado.

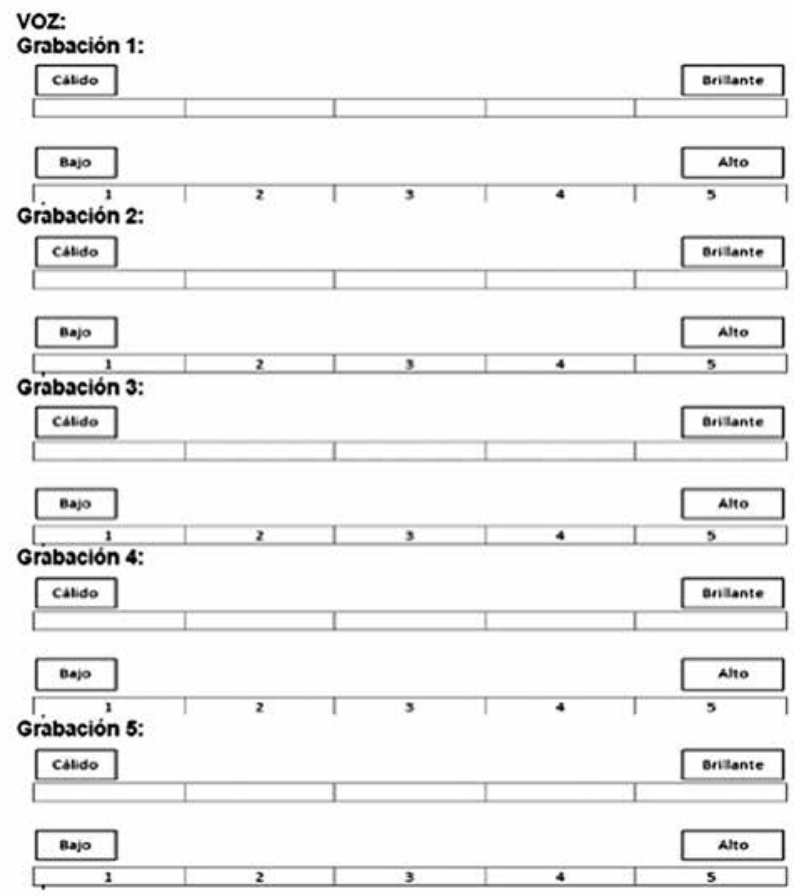

Fig. 5 Aplicación de encuesta para evaluar parámetros subjetivos en muestras de la voz.

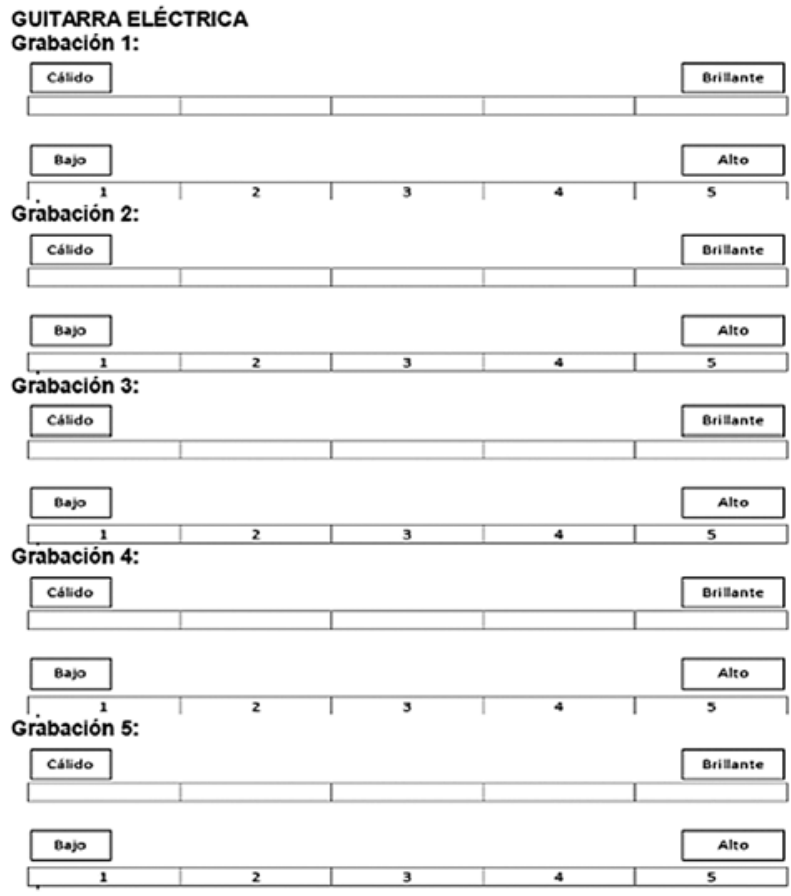

Fig. 6 Aplicación de encuesta para evaluar parámetros subjetivos en muestras de la guitarra eléctrica. 
Así mismo, se entrevista verbalmente a los encuestados, con el fin de que expresen lo percibido en cada una de las grabaciones, para encontrar, de esta manera, relaciones entre los datos cuantitativos y cualitativos y poder realizar comparaciones directas entre los parámetros objetivos, la calidez y el brillo.

\section{B. Población y Muestra}

Al contar con dos clases de pruebas para evaluar la calidad de cada uno de los diferentes preamplificadores son esenciales dos clases de muestras. La primera son los amplificadores de audio frecuencia de señal pequeña, en los que las muestras se reducen a tres dispositivos: API 512C, Manley Voxbox, banco de pre-amplificadores en sus tres configuraciones.

La segunda parte contará con la población de ingenieros de sonido con experiencia entre 15 y 26 años en el área de grabación. Para esto se aplicará la encuesta a una muestra no probabilística de expertos Hernández, Fernández \& Baptista, [4]), conformada por cuatro ingenieros:
Ing. Mauricio Cano, 26 años de experiencia. Ing. William Romo, 20 años de experiencia. Ing. Andrés Landinez, 17 años de experiencia. Ing. Francisco Castro, 15 años de experiencia.

\section{Desarrollo Ingenieril}

La Fig. 7 muestra el diagrama de bloques del banco de pruebas desarrollado para la evaluación de los parámetros anteriormente mencionados. La entrada del sistema es una señal procesada a través de cada uno de los DUT (Dispositivo Bajo Prueba) que para este caso son cinco (5) dispositivos diferentes: DUT 1 es el pre-amplificador de estado sólido API 512C; DUT 2 es el pre-amplificador de válvulas al vacío Manley Voxbox; DUT 3 es la primera configuración del dispositivo construido BP (banco de pre-amplificadores) cuya salida es tomada de una etapa de tubos de cátodo seguidor; DUT 4 es el BP en su segunda configuración haciendo uso del transformador 1 (TRIAD HS-50) y DUT 5 es el BP en su tercera configuración haciendo uso del transformador 2 (Hammond 850NA).

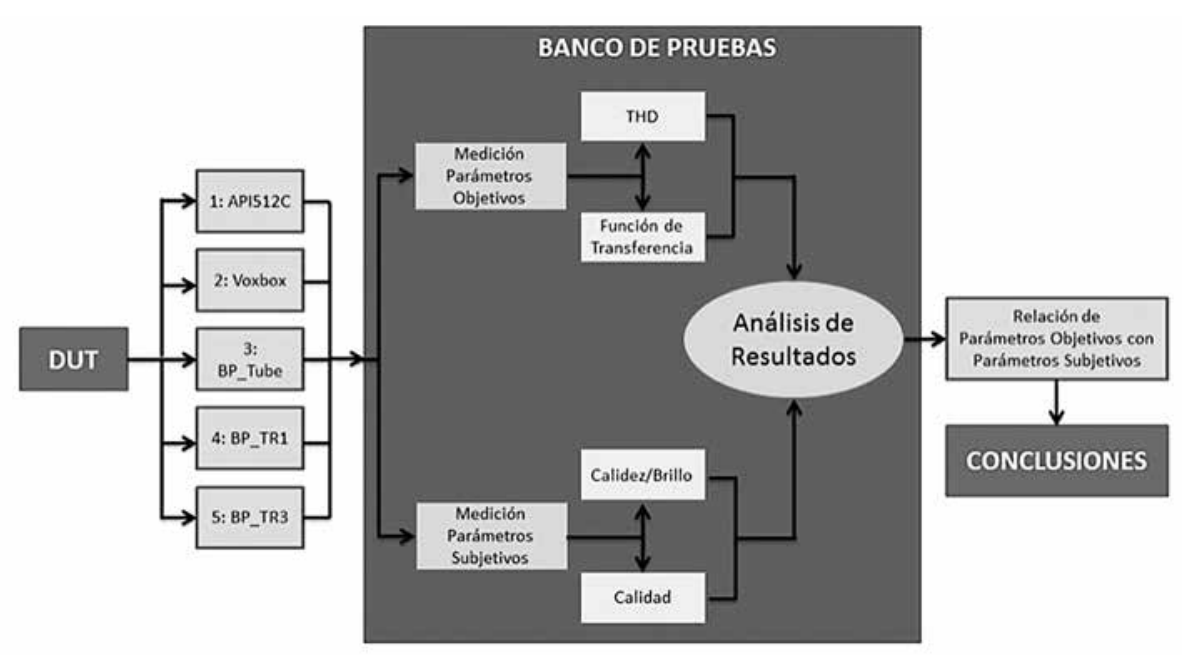

Fig. 7 Diagrama de bloques del banco de pruebas desarrollado .

Al ingresar la señal de salida de cada uno de los DUT al banco de pruebas, estas son sometidas a las mediciones de parámetros objetivos (THD -Análisis de Fourier- y función de transferencia) y a las mediciones de parámetros subjetivos (calidez/brillo y calidad) para realizar un análisis de resultados que finalmente permita relacionar ambos tipos de parámetros identificando las características que imprime el circuito de amplificación sobre la señal de entrada, que logran ser percibidas auditivamente y calificadas con alguno de los parámetros subjetivos evaluados [5]. 


\section{Análisis de Resultados}

\section{A. Análisis de parámetros objetivos.}

1) Función de Transferencia. Después de haber realizado las respectivas mediciones de cada uno de los DUT, se comparan los resultados de función de transferencia normalizados a $0 \mathrm{~dB}$ como se muestra en la Fig. 8.

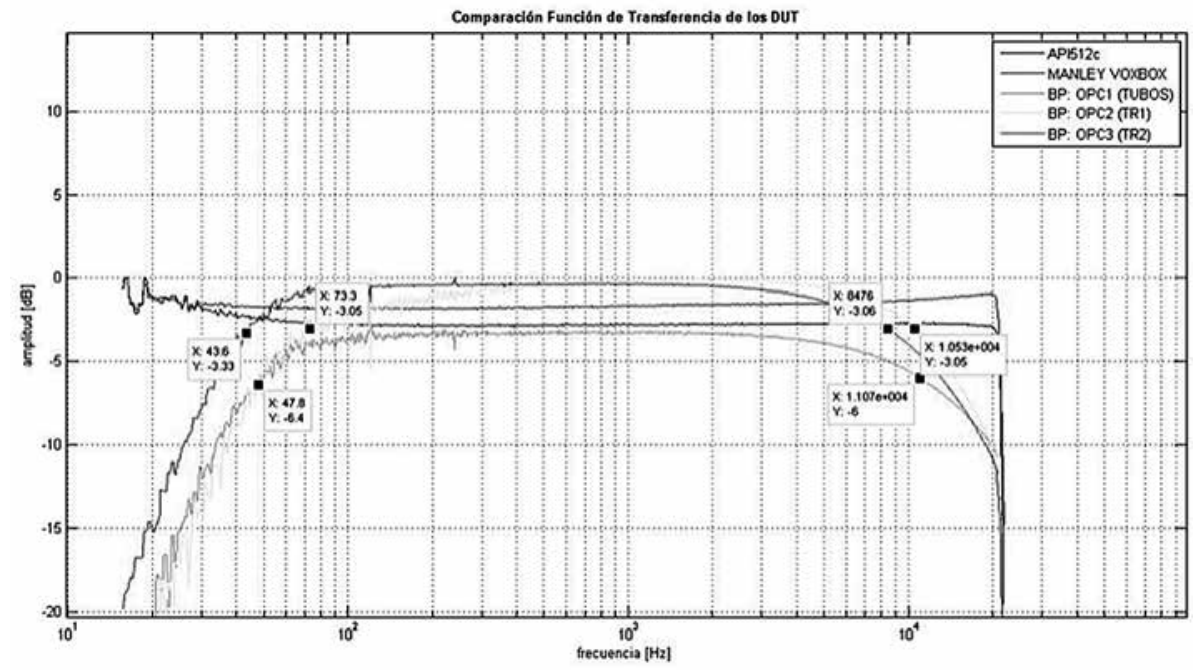

Fig. 8 Función de Transferencia de los DUT. Amplitud [dB] vs Frecuencia [Hz]

El pre-amplificador API 512c (azul) muestra un comportamiento plano desde los $70 \mathrm{~Hz}$ hasta los $20 \mathrm{KHz}$ (incluso donde permite analizar la frecuencia de muestreo). Se puede observar también que se presenta un incremento en bajas frecuencias de $70 \mathrm{~Hz}$ hacia abajo.

En el caso del Manley Voxbox (rojo), la respuesta es plana desde los $20 \mathrm{~Hz}$ (descartando el rizado ocasionado por la respuesta del software), hasta los $4 \mathrm{KHz}$ y de ahí en adelante empieza a haber un incremento en altas frecuencias hasta los $20 \mathrm{KHz}$.

El banco de pre-amplificadores presenta para sus tres configuraciones un rizado en bajas frecuencias que se extiende hasta los $300 \mathrm{~Hz}$ ocasionado por la fuente de alimentación.

La primera configuración del banco de pre-amplificadores (tubos-verde) presenta una respuesta plana que va desde los $47 \mathrm{~Hz}$ hasta los $10 \mathrm{KHz}$ y de ahí en adelante hay un decremento en la amplitud.

La segunda configuración (transformador 1amarillo) presenta una respuesta plana a partir de los $73 \mathrm{~Hz}$ hasta los $10 \mathrm{KHz}$ y posterior a esto decae de manera similar a la configuración uno.

La tercera configuración (transformador 2- magenta) responde de manera plana partiendo de los $43 \mathrm{~Hz}$ hasta los $8.5 \mathrm{KHz}$ y de ahí en adelante empieza a decaer.

Para hacer posible la comparación entre las fases de cada DUT, se realizó primero la exclusión de la fase inicial del sistema de medición efectuando una resta entre las fases de cada DUT y la fase del sistema de medición con el fin de encontrar la respuesta en fase de cada dispositivo y así obtener la comparación de la Fig. 9. 


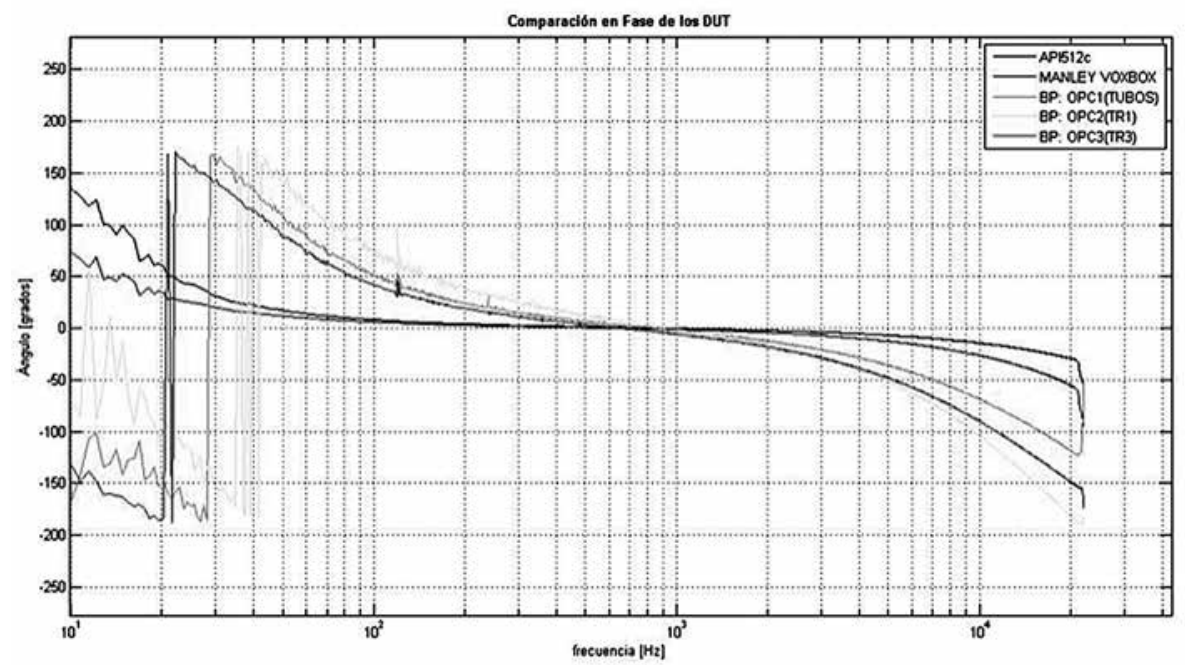

Fig. 9 Respuesta en fase de los DUT, Ángulo [Grados] vs Frecuencia [Hz]

El comportamiento del pre-amplificador API (azul) y el Manley (rojo) es lineal entre $20 \mathrm{~Hz}$ $20 \mathrm{KHz}$; sin embargo, en las bajas frecuencias se observa que hay mayor linealidad en el API y para las frecuencias altas ocurre lo contrario: el API se comporta más plano que el Manley, partiendo de los $12 \mathrm{KHz}$.

Las configuraciones del banco de pruebas tienen un comportamiento similar entre sí. La curva de fase no presenta el comportamiento lineal de los dos dispositivos anteriores (API y Manley) y el corrimiento máximo de fase (180 grados) se encuentra en $30 \mathrm{~Hz}$ para la primera configuración, en $40 \mathrm{~Hz}$ para la segunda y en $20 \mathrm{~Hz}$ para la tercera. A partir de los $2 \mathrm{KHz}$, el de mayor corrimiento en la fase es la segunda configuración del BP seguido de la opción tres y por último la opción uno. Esto debido a que las dos últimas opciones tienen transformadores con inductancias que alteran el comportamiento del tubo empleado.

2) THD -Análisis de Fourier-. Para analizar los resultados obtenidos en esta medición y poder comparar la distorsión armónica de todos los DUT, se consideraron los diez primeros armónicos y se determinó la amplitud relativa a la frecuencia fundamental para cada uno de los dispositivos, buscando determinar qué tan presente está cada Armónico [7]. La tabla I relaciona los niveles en dB de cada armónico (2-10) con cada uno de los dispositivos:

TABLA I

Niveles Relativos de los 10 Primeros de los Dut Respecto a la Fundamental

\begin{tabular}{|c|c|c|c|c|c|c|}
\cline { 2 - 7 } \multicolumn{1}{c|}{} & Generador & API512c & MANLEY & $\begin{array}{c}\text { BP: } \\
\text { TUBOS }\end{array}$ & BP: TR1 & BP: TR2 \\
\hline $\begin{array}{c}\text { No. De } \\
\text { Armónico }\end{array}$ & $\begin{array}{c}\text { Amplitud } \\
\text { [dB] }\end{array}$ & $\begin{array}{c}\text { Amplitud } \\
\text { [dB] }\end{array}$ & $\begin{array}{c}\text { Amplitud } \\
\text { [dB] }\end{array}$ & $\begin{array}{c}\text { Amplitud } \\
\text { [dB] }\end{array}$ & $\begin{array}{c}\text { Amplitud } \\
\text { [dB] }\end{array}$ & $\begin{array}{c}\text { Amplitud } \\
\text { [dB] }\end{array}$ \\
\hline $\mathbf{2}$ & $-45,15$ & $-45,21$ & $-53,82$ & $-41,89$ & $-23,7$ & $-36,2$ \\
\hline $\mathbf{3}$ & $-38,76$ & $-37,09$ & $-30,88$ & $-52,25$ & $-27,92$ & $-37,89$ \\
\hline $\mathbf{4}$ & $-52,95$ & $-54,8$ & $-48,58$ & $-64,19$ & $-39,21$ & $-39,83$ \\
\hline $\mathbf{5}$ & - INF & - INF & $-50,3$ & $-78,21$ & $-41,12$ & $-43,83$ \\
\hline $\mathbf{6}$ & $-56,17$ & $-58,01$ & $-51,54$ & $-78,98$ & $-42,47$ & $-50,05$ \\
\hline $\mathbf{7}$ & $-59,07$ & $-55,98$ & $-47,47$ & $-73,02$ & $-55,76$ & $-56,92$ \\
\hline $\mathbf{8}$ & $-85,32$ & $-82,77$ & $-61,61$ & $-88,47$ & $-52,12$ & $-57,73$ \\
\hline $\mathbf{9}$ & $-53,53$ & $-50,8$ & $-41,46$ & $-68,52$ & $-60,67$ & -60 \\
\hline $\mathbf{1 0}$ & $-70,38$ & $-70,65$ & $-61,82$ & - INF & $-62,45$ & $-60,62$ \\
\hline
\end{tabular}


Partiendo de los datos de la tabla I, se halla la diferencia del nivel de cada armónico con respec- to al nivel de la frecuencia fundamental, obteniendo los resultados ilustrados en la Fig. 10.

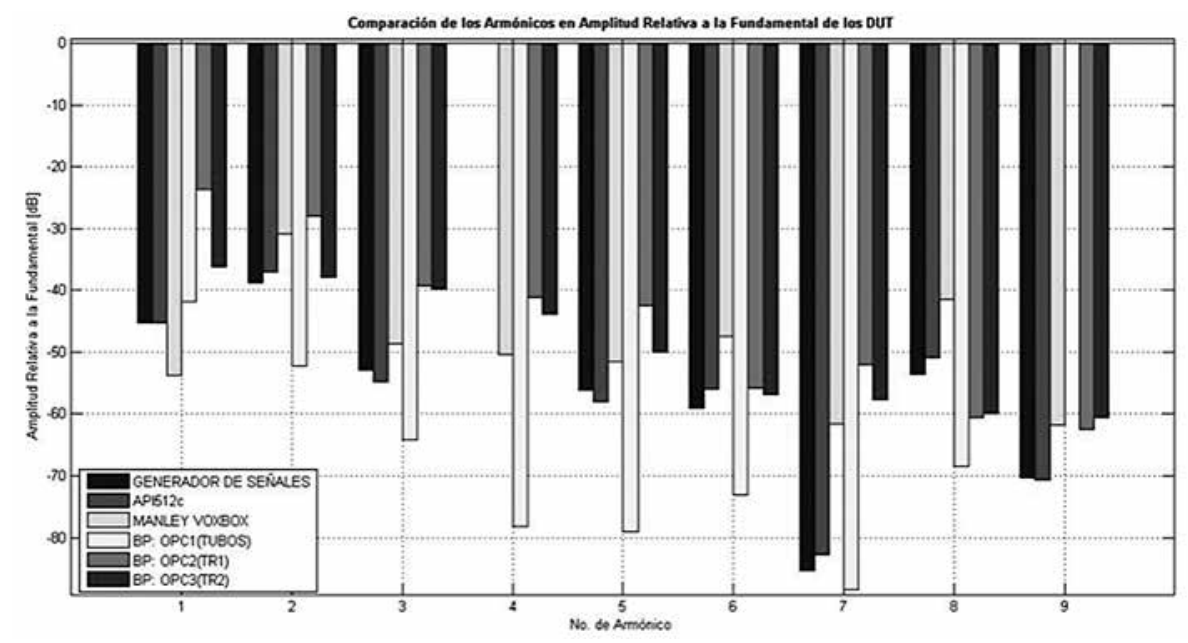

Fig. 10 Comparación de los Armónicos en Amplitud Relativa a la Fundamental de los DUT, No. de Armónico Vs Amplitud Relativa a la Fundamental [dB]

En la Fig. 10, los armónicos que se acercan a $-100 \mathrm{~dB}$ son los que poseen menor presencia en el Análisis de Fourier. El azul más oscuro representa los armónicos del generador implementado para la medición y el azul más claro los del API 512c. Como se observa, los armónicos del API son bastante similares a los del generador y están presentes casi al mismo nivel, permitiendo inferir que este primer DUT es bastante limpio y no aporta a la señal un contenido armónico muy distinto a sus propiedades originales.

En cuanto a los otros dispositivos, se puede observar que la presencia de armónicos difiere del generador; esto quiere decir, que sí imprimen en la señal un contenido armónico diferente a sus características iniciales. Se ve, por ejemplo, que en el primer armónico, el DUT que posee mayor presencia es el BP en la configuración TR1; aproximadamente a $10 \mathrm{~dB}$ de diferencia se encuentra el BP en la configuración TR2, seguido por el BP en la configuración tubos y se observa que el Manley posee una presencia en este armónico, incluso menor, que la del generador.

En el segundo armónico, el de mayor presencia es de nuevo el BP-TR1 pero esta vez de menor nivel que en el armónico anterior; el BP-TR2 se presenta casi al mismo nivel que en el primer armónico y Manley aparece más presente que el generador. El BP-Tubos se encuentra en cambio menos presente que el API y el generador.

El tercer armónico cuenta con una menor presencia de todos los DUT y del generador. El BP-TR1 se encuentra casi al mismo nivel que el BP-TR2, los cuales están por encima de los demás dispositivos, y el BP-Tubos es el de menor presencia.

En el cuarto armónico no hay presencia del API. El BP-TR1 es el más presente y el BP-Tubos el menos presente. En el quinto armónico vuelve a haber presencia del API (y del generador) y se repite el siguiente orden del más presente al menos presente: BP-TR1, BP-TR2, Manley, API y BP-Tubos.

En el sexto armónico aparece el Manley como el más presente y el BP-Tubos como el menos; en el séptimo armónico el BP-TR1 vuelve a ser el más presente, seguido por el BP-TR2 y el BPTubos es de nuevo el de menor presencia.

En el octavo armónico el Manley es el más presente, seguido por el API y posteriormente por el BP-TR2; el BP-Tubos es el menos presente. El noveno armónico cuenta con mayor presencia del 
BP-TR2, seguido por el Manley, posteriormente, por el BP-TR1, luego el API y, finalmente, el BP-Tubos que posee un nivel por debajo de los $-100 \mathrm{~dB}$.

\section{B. Análisis de parámetros subjetivos.}

Partiendo de los datos obtenidos en las encuestas (estos datos son de uso de los investigadores según el consentimiento informado a los encues-

Promedio Aritmético de los Parámetros Calidez/Brillo y Calidad, Covarianza y Correlación de los Datos Obtenidos en Prueba No Probabilística de Expertos

\section{VOZ}

\begin{tabular}{|c|c|c|c|c|c|c|c|c|c|c|}
\hline & \multirow{2}{*}{\multicolumn{2}{|c|}{ API }} & \multirow{2}{*}{\multicolumn{2}{|c|}{ Manley }} & \multirow{2}{*}{\multicolumn{2}{|c|}{ BP-Tube }} & \multirow{2}{*}{\multicolumn{2}{|c|}{ BP-TR1 }} & \multirow{2}{*}{\multicolumn{2}{|c|}{ BP-TR2 }} \\
\hline & & & & & & & & & & \\
\hline & Cal-Bril & Calid & Cal-Bril & Calid & Cal-Bril & Calid & Cal-Bril & Calid & Cal-Bril & Calid \\
\hline PROM & 2,75 & 3 & 2,75 & 3,75 & 1,75 & 3,5 & 1,75 & 2,75 & 1,5 & 3,25 \\
\hline COVARIANZA & \multicolumn{2}{|c|}{0,25} & \multicolumn{2}{|c|}{$-0,06$} & \multicolumn{2}{|c|}{$-0,13$} & \multicolumn{2}{|c|}{0,19} & \multicolumn{2}{|c|}{$-0,13$} \\
\hline CORRELACION & \multicolumn{2}{|c|}{0,82} & \multicolumn{2}{|c|}{$-0,17$} & \multicolumn{2}{|c|}{$-0,26$} & \multicolumn{2}{|c|}{0,52} & \multicolumn{2}{|c|}{$-0,58$} \\
\hline
\end{tabular}

GUITARRA ELECTRICA

\begin{tabular}{|c|c|c|c|c|c|c|c|c|c|c|}
\hline & \multicolumn{2}{|c|}{ API } & \multicolumn{2}{|c|}{ Manley } & \multicolumn{2}{|c|}{ BP-Tube } & \multicolumn{2}{|c|}{ BP-TR1 } & \multicolumn{2}{|c|}{ BP-TR2 } \\
\hline & Cal-Bril & Calid & Cal-Bril & Calid & Cal-Bril & Calid & Cal-Bril & Calid & Cal-Bril & Calid \\
\hline PROM & 3,75 & 3,25 & 2,75 & 3,75 & 2,25 & 3,75 & 2 & 4 & 1,75 & 3,75 \\
\hline \multirow{2}{*}{$\begin{array}{l}\text { COVARIANZA } \\
\text { CORRELACION }\end{array}$} & \multicolumn{2}{|c|}{0,31} & \multicolumn{2}{|c|}{$-0,81$} & \multicolumn{2}{|c|}{0,44} & \multicolumn{2}{|c|}{0,56} & \multicolumn{2}{|c|}{0,06} \\
\hline & \multicolumn{2}{|c|}{0,57} & \multicolumn{2}{|c|}{$-0,90$} & \multicolumn{2}{|c|}{0,59} & \multicolumn{2}{|c|}{0,52} & \multicolumn{2}{|c|}{0,13} \\
\hline
\end{tabular}

A pesar de que la muestra es bastante reducida, en la mayoría de los casos la correlación se presenta por encima de 0.5 tanto hacia el lado de una correlación positiva indicando una relación de pendiente positiva entre ambas variables (Calidez/Brillo y Calidad), como hacia el lado negativo que indica una pendiente negativa en la relación de ambas.

Los datos recolectados en la encuesta, muestran que en el parámetro de calidez y brillo hay resultados similares para los cuatro ingenieros entrevistados, quienes coincidieron en cuál es el pre-amplificador más brillante y cuál es el más cálido. Es decir, que determinando el promedio entre las apreciaciones realizadas para la voz y la guitarra, es posible ordenar los pre-amplificadores testeados del más cálido al más brillante como se muestra en la tabla III. tados, por lo tanto, solo los investigadores tienen acceso a esta tabulación), se calcula el promedio aritmético de cada parámetro evaluado, así como, la covarianza y la correlación entre ambos parámetros para determinar qué tanto se relacionan entre ellos [8]. Estos datos se ilustran en la tabla II.

TABLA II 
composición musical, el color de la voz del intérprete y el tipo de distorsión en la guitarra del solo, entre otros.

Los siguientes son los testimonios de las entrevistas verbales a los ingenieros refiriéndose a cada una de las grabaciones:

En general, la grabación 1 (API 512c) fue considerada brillante. I ingeniero Mauricio Cano afirmó en su entrevista que "En la voz me pareció el API un poquito brillante..... El ingeniero William Romo afirmó: "Yo escuché algo de la voz como lo que uno buscaría al ecualizar, en el primero (API 512c), o sea como buscándolo más brillante, buscaría ese punto... En las guitarras el primero es más brillante...”. El ingeniero Francisco Castro coincidió: “...sí, el API era ahí como súper rápido y aparecían las Ss ahí mismo y súper descansado...". Finalmente, el ingeniero Andrés Landinez afirmó que: "En cuanto a la guitarra eléctrica pues sí un color bastante no brilloso sino chillón, sobre todo en las primeras muestras; en la últimas ya no tanto pero no dejaba de ser chillón...”.

Estas apreciaciones sobre el pre-amplificador API, permiten concluir que su marcada presencia en las Ss, no es muy favorable para la voz. El exceso de brillo hace que la muestra pierda naturalidad y fue puntuada por los cuatro ingenieros como la de menor preferencia para este instrumento [9]. En cuanto a la guitarra, ocurrió algo similar y obtuvo también el promedio más bajo en el parámetro de calidad. La grabación 2 (Manley Voxbox) generó las siguientes reacciones: el ingeniero Mauricio Cano consideró que estaba un poquito brillante; el ingeniero William Romo afirmó que en la grabación 2 los brillos bajan un poco y las siguientes se vuelven más naturales; el ingeniero Francisco Castro, por su parte, afirmó que: “....ahora, el Manley es un muy buen pre, se escucha no solo bonito, sino como musical, abierto, es como debería sonar la música, grande, tapada de armónicos...”.

En general, el pre-amplificador Manley fue el preferido para la voz con relación al promedio; sin embargo, no fue elegido por todos como el mejor. En cuanto a la guitarra, no hay una preferencia tan marcada por ser apreciaciones tan subjetivas.Los criterios que llevan a preferir una grabación más que otra, están relacionados con elementos como la naturalidad del sonido como lo afirma el ingeniero William Romo: “...En las guitarras el primero es más brillante, el segundo menos, el tercero ya bajaron un poquito, se vuelven más cálidos obviamente, que es lo que uno busca a veces, la naturalidad, yo busco más la naturalidad del instrumento. Si cuando tú estás hablando tu no ves una parte muy brillante, solo cuando tú vas a procesarlo ya toca sacarlo un poco porque hay que sacarlo respecto a los demás instrumentos...".

Las grabaciones 3, 4 y 5, correspondientes al BP en sus respectivas configuraciones, están calificadas como las de mayor calidez. Sin embargo, esto no garantiza que sean las preferidas, hubo resultados dispersos: el ingeniero Mauricio Cano afirmó que "...El de ustedes (BP) tiene un toque de brillo que a mí particularmente me gusta, o sea no es el chillón ni es sin, sino que está ahí, rico con las Ss bien, sin exagerar. No hay que moverlo, no hay que mover mucho..." El ingeniero William Romo sostuvo que "...O sea por naturalidad escuché ese más dulce (el BP); ahora, hay unos que les gusta escuchar algo brillante, escogen lo otro pero ya depende, pero yo lo puedo recuperar en una ecualización posterior...".

Por otro lado, el ingeniero Francisco Castro manifestó que "Este transformador que escogieron (grabación 4) me parece que es súper resonante, cero musical en mi concepto, les puede decir alguien otra cosa, acá dije que estaba alto y la respuesta en bajas frecuencias no...".

Lo dicho por los ingenieros evidencia que una coloración que puede favorecer a un instrumento, puede no ser tan necesaria en el otro y que las características propias del pre-amplificador puede que aporten a cierta señal como afirma el ingeniero Francisco Castro: “... (Con respecto a la muestra 5) a la guitarra seguro le faltaba algo y este se lo aportaba..... 
En cuanto a promedio aritmético, el preferido para la guitarra fue el BP-TR2 correspondiente a la grabación 4, revelando que a pesar de analizar las señales a través de los mismos dispositivos, para un instrumento se puede ajustar mejor uno y para el otro instrumento algo distinto siempre buscando que suene musical y favorezca a la composición.

\section{Conclusiones}

Al relacionar los parámetros objetivos medidos, con las apreciaciones subjetivas obtenidas de la muestra no probabilística de expertos se puede inferir lo siguiente:

1) El hecho de que el DUT1 (API512c) haya sido considerado el más brillante, está relacionado con que este dispositivo presenta una respuesta en frecuencia plana en el rango audible como se observa en la Fig. 8 y su comportamiento en los nueve primeros armónicos, corresponde a la distorsión de la señal entrante (generador de señales) como se observa en la Fig. 10, lo que indica que este DUT es el que la conserva con mayor fidelidad con una diferencia no mayor de $3 \mathrm{~dB}$ en cada armónico (Tabla I).

2) En cuanto al DUT2 (Manley Voxbox), el ingeniero Francisco Castro afirmó que "se escucha no solo bonito, sino como musical, abierto, es como debería sonar la música, grande, tapada de armónicos". Esta afirmación coincide con la contribución de este DUT en los nueve primeros armónicos analizados, en los que se observa (Fig. 10) que hay mayor presencia en comparación con el nivel de los armónicos de la fuente (generador de señales) y con su respuesta en frecuencia que presenta un realce a partir de los $12 \mathrm{KHz}$ como revela la Fig. 8.

3) EI DUT 3 en su primera configuración (BP-Tubos) fue percibido como cálido con un promedio de 2.00 que se puede asociar con la caída que presenta en su respuesta en frecuencia después de los $11 \mathrm{KHz}$, sumada a la presencia de nivel en los ocho primeros armónicos (Fig.10). Esta configuración del BP, fue percibida como natural por el ingeniero William Romo. Esta naturalidad se asocia con que a pesar de que hay presencia de amplitud en todos los armónicos analizados, esta es de menor nivel con respecto a las otras configuraciones del DUT3 y los DUT1 y 2 como indica la Fig. 10.

4) La segunda configuración del BP (BP-TR1), DUT 4, fue calificada como cálido con un promedio de 1.88 que se relaciona con la respuesta en frecuencia que presenta un decremento a partir de los $10 \mathrm{KHz}$. Su contribución en los cinco primeros armónicos es la más elevada con respecto a los demás dispositivos analizados y esto se refleja en los resultados de calidad obtenidos en la muestra de expertos, ya que la grabación 4 fue elegida como la menos favorita con un promedio de 2,75 , mientras que en la guitarra obtuvo un promedio de 4 , lo que la convierte en la preferida por encima de las otras grabaciones. Es posible deducir entonces que como la muestra de la guitarra presentaba distorsión de amplificador, la fuerte presencia de los armónicos mencionados favoreció al instrumento. En cambio, en la voz ocurrió lo contrario debido a que la fuente debe preservarse limpia en términos de distorsión.

5) El DUT 5, la tercera configuración del BP (BP-TR2), fue calificada como la de mayor calidez con un promedio de 1,63 que se relaciona con el corte en su respuesta en frecuencia a partir de los $8.5 \mathrm{KHz}$, la cual se encuentra por debajo de los cortes en altas frecuencias de las dos primeras configuraciones. Es decir, que entre más abajo se presentó el corte, el DUT fue considerado de mayor calidez.

En general se puede afirmar que los parámetros subjetivos de calidez y brillo responden a una combinación entre la respuesta en frecuencia del dispositivo y su contribución en los armónicos, relacionando los aspectos electrónicos del circuito con lo que se percibe auditivamente en la señal procesada. No se descarta la posibilidad de que estén también asociados a otros parámetros electrónicos como la respuesta al transiente [10]. 
Después de analizar todos los parámetros mencionados, es posible resaltar que los dispositivos que emplean válvulas al vacío en su circuito de amplificación, sí se perciben como cálidos y presentan una contribución particular en los armónicos que no es propia de la señal de entrada y difiere en gran medida de las contribuciones del pre-amplificador de transistores analizado.

\section{REFERENCIAS}

[1] E. Baarbour, The cool sound of tubes, IEEE Spectrum, 1998.

[2] Andrzej, Dobrucki, M. Stanislaw and K. Maurycy, Subjective and objective evaluation of the audio vacuum-tube amplifiers. AES Convention Paper 7806, 2005.

[3] B. McCarthy, Sound systems design and optimization, Focal Press, 2007.

[4] R. Hernández, C. Fernández y P. Baptista, Metodología de lainvestigación, 2da. ed., 1998. 